\title{
Sialolithiasis der Gl. submandibularis: Bei großen Konkrementen drüsenerhaltend operieren
}

leine Steine kann der Operateur meist ohne große Komplikationen entfernen. Doch auch große Konkremente können ohne Verlust der Drüse extrahiert werden. Dr. Nils Klintworth, Universität Erlangen, machte darauf aufmerksam, dass sich in Abhängigkeit von Größe, Anzahl und Lokalisation von Steinen der Gl. submandibularis mehrere Optionen anbieten. Dazu zählen endoskopische Entfernung, Speichelgangschlitzung sowie die extrakorporale Stoßwellenlithotripsie (ESWL). Die Drüse sollte nur in Ausnahmefällen primär reseziert werden. Zwischen 2003 und 2009 waren in der HNO-
Klinik der Universität Erlangen insgesamt 730 Konkremente der Gl. submandibularis entfernt worden. Klintworth präsentierte klinische Ergebnisse von 73 dieser Steine, die alle über $15 \mathrm{~mm}$ groß waren.

Die Speichelgangsendoskopie konnte bei den großen Konkrementen nicht durchgeführt werden. Eine ESWL war nur in einem Fall möglich. 70 Steine (96\%) entfernten die HNO-Ärzte über eine transorale Speichelgangschlitzung. Lediglich bei zwei Patienten war eine primäre Entfernung der Drüse notwendig.

In 77\% der Fälle nach Gangschlitzung waren die Patienten komplett „steinfrei“.

\section{Elektive HNO-Eingriffe: Patienten zeigen keine erhöhte MRSA-Prävalenz}

roblemkeime wie der multiresistente
Staphylococcus aureus (MRSA) werden weltweit mit erhöhter Aufmerksamkeit beobachtet. HNO-Patienten, die sich einer elektiven Operation unterziehen, scheinen aber nicht mehr gefährdet als die Allgemeinbevölkerung.

Dr. Andreas Eckert vom Helios-Klinikum Berlin-Buch, machte auf die Daten des Robert-Koch-Instituts aufmerksam. Danach lag die MRSA-Prävalenz der Bevölkerung im Raum Südbrandenburg bei 0,77\% [Epidemiol Bull 2012; 8: 63-67]. Er verwies auf die strikte Regelung im Nach- barland Holland, nach der alle stationären Patienten solange in Isolation bleiben, bis sich der bei Aufnahme entnommene Abstrich als negativ erwiesen hat. Dieses Vorgehen wäre auf die Bundesrepublik nicht übertragbar, so Eckert; die Niederlande seien aber eines der Länder mit der niedrigsten MRSA-Prävalenz.

Eckert stellte eine Untersuchung mit insgesamt 473 Patienten vor, die wegen eines elektiven HNO-Eingriffs aufgenommen wurden. Bei allen Studienteilnehmern entnahmen die Ärzte Abstriche aus Nasenvorhof und Rachen. Bei fünf
Bei den übrigen Fällen verblieben Konkremente bzw. Konkrementreste; nur ein Patient wies dabei Beschwerden auf. Nach einer mittleren Nachbeobachtungszeit von drei Jahren waren 96\% der untersuchten Patienten aktuell beschwerdefrei. Beschwerden im Zeitraum ab der Operation waren bei $23 \%$ aufgetreten. In $16 \%$ der Fälle war es zum Rezidiv gekommen.

Auch bei großen Konkrementen über $15 \mathrm{~mm}$, so Klintworth, sei eine drüsenerhaltende Operation fast immer möglich. Der Patient sollte über die therapeutischen Möglichkeiten, aber auch über die Möglichkeit eines Rezidivs aufgeklärt werden.

Dr. Andreas Fischer

Klintworth N.: Drüsenerhaltende Therapie bei großen Konkrementen der GI. Submandibularis

Patienten wurde ein MRSA neu diagnostiziert. Vier dieser Patienten wiesen ein entsprechendes Risikoprofil auf (Heimpatienten, Aufenthalte auf einer Intensivstation). Nur in einem Fall handelte es sich um eine Nicht-Risikopatientin.

Damit ergab sich eine Prävalenz von 0,21\% bei "gesunden“ HNO-Patienten ohne erhöhtes Risiko. Ein intensiviertes MRSA-Screening mit Abstrich und Isolation bei positivem Befund sei damit weiterhin nur für Patienten mit erhöhtem Risikoprofil indiziert, schloss Eckert.

Dr. Andreas Fischer

Eckert A: Multiresistenter Staphylococcus aureus (MRSA) - sind in der HNO nur Risikopatienten besiedelt?

\section{Hier steht eine Anzeige.}

\author{
望 Springer
}

